Artigos

\title{
Gabriela del Valle Vergara
}

Universidad Nacional de Villa María, Villa María, Córdoba, Argentina Consejo Nacional de Investigaciones Científicas y Técnicas, Argentina

\section{Cuerpos, sensibilidades y acción colectiva (Argentina, 2002)}

Resumen: El artículo analiza las relaciones entre condiciones de vida, sensibilidades y acciones, para comprender las formas en que el conflicto puede (o no) emerger. Para ello, se utilizan datos secundarios provenientes de Latinobarómetro, en 2002, año identificado con una de las últimas crisis más importantes de Argentina y se comparan mujeres y varones asalariados y cuentapropistas. $\mathrm{Del}$ análisis surge que hay inacción bajo dos lógicas diferentes pero complementarias: individualismo/solidarismo e individualismo/soportabilidad. Esta aproximación cuantitativa no pretende agotar las relaciones entre conceptos y variables propuestos, sino alentar las posibilidades de comprensión de la estructuración capitalista actual.

Palabras clave: cuerpos; sensibilidades; conflicto; género; trabajo

\begin{abstract}
"Hace cinco meses estábamos desocupadas y de pronto nos encontramos con esta oportunidad que no queremos desaprovechar (...) Hemos aprendido algo y eso nos hace sentir muy bien, nos hace sentir útiles otra vez (...) Se puede...sabemos que se puede. No hay que quedarse encerrada en medio de cuatro paredes con los brazos cruzados, esperando una ayuda. Tenemos manos, brazos y ganas de trabajar y tenemos también la necesidad de buscar nuestro propio sustento para llevarle respuestas a nuestros hijos", (entrevista a Rosa Tolosa, presidenta del grupo cooperativo "Sueños Verdes", Diario La Voz del Interior, 9 de julio de 2002)
\end{abstract}

\section{Introducción'}

Capitalismo, cuerpos y sensibilidades se han articulado de manera diversa y compleja según la (nueva) división internacional del trabajo, el neocolonialismo, el imperialismo y la dependencia.

La cita del epígrafe nos invita - más allá de una mirada miserabilista - a indagar cómo se traman las percepciones, las prácticas y las posibilidades de construcción colectiva

${ }^{1}$ Este artículo es parte del proyecto "Sensibilidades en tensión: Trabajos, acciones colectivas y mujeres. Un análisis desde la estructuración social en San Francisco y Villa María (2002-2016)" aprobado por CONICET. 
de futuros. El camino desde la desocupación, el sentirse inútil, encerrada con los brazos cruzados hacia el movimiento de partes - fragmentadas y escindidas - del cuerpo (manos, brazos, ganas) que ven una oportunidad que hace sentir bien, parece tener mucho que ver con las formas en que opera el capitalismo neocolonial dependiente en el siglo XXI. Sus principales características son la depredación, la destrucción, el despliegue de formas de sometimiento humano/de la naturaleza, desde la esclavitud, el turismo sexual, la prostitución, hasta la venta de órganos o partes del cuerpo (David HARVEY, 2004; Mariarosa DALLA COSTA, 1994).

Pero además, el capitalismo como aparato basado en la expropiación de energías corporales y sociales despliega otros mecanismos que permiten su naturalización (Lùc BOLTANSKI y Eve CHIAPELLO, 2002). Creencias, fantasmas y fantasías sociales contribuyen a instalar el miedo, anhelar un futuro mejor, o esperar pasivamente, porque es lo único que se puede hacer. La presencia de dispositivos que regulan sensaciones y permiten soportar las injusticias advierte acerca de sutiles y silenciosas formas que operan en y a través de los cuerpos/emociones, en las porosas urdimbres de la vida cotidiana. Las desconexiones entre percepciones, emociones y prácticas se comprenden a partir del funcionamiento ideológico que Marx identificó cuando el trabajador no percibía que muchos otros se encontraban en una situación similar. ${ }^{2}$

Es decir, no siempre las relaciones son lineales, en el sentido de que si la gente dice estar feliz se debe a que hay progreso en las sociedades (Ruut VEENHOVEN, 2005) o que, ante medidas económicas de ajuste, hay conflicto por las broncas e insatisfacciones que genera el capitalismo (Tova BENSKI y Lauren LANGMAN, 2013). Las tramas que se tejen entre sociedad, clases, géneros, etnias y sensibilidades desafían a esgrimir nuevas hipótesis, dado que la "ausencia de conflicto" podría significar una mirada miope, sensu Melucci, acerca del estado de estructuración capitalista.

En este marco, se presenta el análisis, conexión e interpretación deposiciones sociales, sensibilidades y prácticas tanto de mujeres como de varones que participaban en el mercado laboral desde el cuentapropismo y el trabajo asalariado, en 2002, ${ }^{3}$ en Argentina.

\footnotetext{
2 Junto a esto, el disfrute desde el consumo es una de las trampas del capitalismo que Marx ya había identificado como recompensa la fuerza de trabajo del obrero (Adrián SCRIBANO, 2013).

${ }^{3}$ El año seleccionado fue escenario de una de las últimas crisis en Argentina, y se corresponde según la información registrada con el año con mayor percepción negativa de la situación del país, de la década. A finales de 2001, Argentina asistió a una crisis institucional con la salida imprevista del por entonces presidente De La Rúa, que se vio acompañada de una crisis económica derivada de la finalización de la convertibilidad cambiaria (que igualaba el valor del peso argentino al dólar estadounidense). Esto elevó los niveles de pobreza por ingreso, redujo los niveles de empleo que se hicieron claramente visibles durante el 2002 Algunas de las expresiones colectivas que se dieron fueron los clubes de trueque, asambleas de vecinos, comedores comunitarios, saqueos. Ahora bien, si se considera a la crisis no solo como un hecho aislado sino como parte de un proceso, el año analizado es el escenario donde recrudecen en un contexto de inestabilidad institucional diferentes focos de conflicto derivados del incremento de pobreza, desempleo, entre otros. El interés por realizar este análisis es, por un lado, ver qué muestran los datos secundarios cuantitativos en relación con las categorías que se ponen en juego. Por otro, tener coordenadas que nos permitan comprender qué pasó con aquella efervescencia que para algunos llegó a ser un momento casi disruptivo o revolucionario. Tal como fue analizado, el periodo posterior da cuenta de que después de la crisis se ha instalado una sensibilidad de la "espera social" (SCRIBANO y Eugenia BOITO, 2010). En otro lugar, hemos analizado (Emilio SEVESO ZANÍN y Gabriela VERGARA MATTAR, 2012), tres dimensiones en las experiencias de actores precarios de Córdoba, que se configuran en el periodo posterior a la crisis de 2001: según su condición socioeconómica se advierte una continuidad, cuando no un empeoramiento, que instala a los cuerpos como puntos inmóviles/ inmovilizados en la pintura del mundo. En cuanto a las capacidades de organización colectiva, presentan un estado de fragmentación que se manifiestan en acciones intermitentes o espasmódicas constituidas en torno a la urgencia y la necesidad. Finalmente, se estructura un horizonte de alternativas que se vive y siente como imposibilidad, a partir del cruce entre dolor y resignación.
} 
A partir de datos secundarios provenientes de Latinobarómetro, se seleccionaron variables que permiten caracterizar aquellas dimensiones. La pregunta que subyace a este mapeo sería indagar las formas por las cuales los agentes sociales en el capitalismo, estando en condiciones de protestar, no lo hacen. Y más concretamente, qué sucede en varones y mujeres, asalariados o cuentapropistas, entre la espera y la protesta. Como hipótesis se sostiene que las sensibilidades, de acuerdo con su geometría corporal, pueden generar desconexiones en las gramáticas de acción, ocluyendo o solapando ciertas características de las condiciones de vida que harían inminente la protesta.

Para ello, se tomará la siguiente estructura argumentativa: 1) se realiza un breve mapeo de los estudios sobre acción colectiva y género; 2) se conceptualizan las categorías de geometría de los cuerpos, gramática de las acciones ${ }^{4}$ y sensibilidades, al tiempo que se mencionan las variables utilizadas para cada uno; 3) se analizan las variables que permiten una aproximación cuantitativa a dichos conceptos, diferenciando a varones y mujeres desde el trabajo asalariado y el cuentapropismo, y 4) se formulan algunas conclusiones provisorias para interpretar las disposiciones y obstáculos a la acción colectiva en términos de una marcada presencia del individualismo que se articula, por un lado, con el solidarismo, y por otro, con la soportabilidad social.

\section{Hacia una revisión de antecedentes}

Dada la vasta producción académica en la temática, este apartado se limita a identificar dos ejes de estudios que se vinculan con este artículo. Por un lado, la presencia de las mujeres en acciones colectivas en general. De los movimientos a las protestas, ha habido conflictos protagonizados por mujeres, propios del trabajo reproductivo, invisibilizado pero imprescindible para la reproducción de la fuerza de trabajo (Silvia FEDERICI, 2013). También, aquellos vinculados con problemáticas que afectan al hogar - tal como la huelga de inquilinos en 1907 - o a los hijos (Mirta LOBATO, 2007; Temma KAPLAN, 1990), pasando por los problemas en el mercado laboral o el sindicato (Gerardo NECOECHEA, 1989), como por los efectos del Ajuste Estructural que inciden en el hogar y moviliza a las mujeres de manera colectiva (Andrea ANDÚJAR, 2007). A esto habría que sumar, los conflictos propios del mercado laboral en el que participan las mujeres (Beatriz CID AGUAYO, 2012).

Las referencias aludidas pueden inscribirse en tres vertientes cuando se visualiza de una manera compleja y heterogénea,el movimiento de mujeres en Latinoamérica. Una de ellas es la que protagonizan las mujeres en los movimientos de derechos humanos; otra, en la que se agrupan mujeres de sectores populares enfrentando consecuencias de los Programas de Ajuste Estructural, y, finalmente, las "mujeres del movimiento feminista, con un fuerte componente en sus orígenes y estabilización de mujeres de sectores medios (Jacquete, 1991; Molyneux, 2001)" (Gabriela DI MARCO, 2010).

Por otro lado, los estudios sobre el lugar de las emociones en las acciones colectivas complejizan la comprensión de las prácticas de los agentes sociales, desde los pioneros aportes de Sorel y Le Bon (Alberto MELUCCI, 1994; Adrián SCRIBANO y Matías ARTESSE, 2012).

Ahora bien, existen al menos dos posibles caminos de indagación que permiten inscribir todas estas movilizaciones, protestas, movimientos sociales en una perspectiva estructural. Por un lado, las acciones colectivas ${ }^{5}$ permiten mapear o radiografiar la estructura

\footnotetext{
${ }^{4}$ En otro lugar (Gabriela VERGARA y Marcelo D'AMICO, 2010) se utilizaron estas categorías para dar cuenta de cómo las percepciones del riesgo incidieron según las geometrías corporales en las acciones que realizaron cuerpos precarios y cuerpos en riesgo de empobrecimiento en 2002, en Córdoba.

${ }^{5}$ Se sigue aquí la propuesta de Scribano (2004) para quien el concepto de acción colectiva abarca a los movimientos sociales, pero también a las protestas, articulando interacciones, negociaciones y conflicto, en el marco de metas, recursos y límites.
} 
conflictual de la sociedad, es decir que desde la acción colectiva se identifica una topología de los conflictos y su proceso de estructuración. La otra forma es ver los conflictos para indagar la acción colectiva, su estado, duración, forma, demandas, etc.

Los movimientos sociales constituyen mensajes que se transmiten a la sociedad y cuestionan lo que pretenden imponer los sistemas tecno-burocráticos, impugnando la racionalidad instrumental propia de los sistemas complejos. En este sentido, "Ios conflictos actúan como señales que nos indican el carácter construido de la acción social y su tendencia a cristalizar en «estructuras» $y$ «sistemas»" (MELUCCI, 1984, p.127).

En el caso de los movimientos de mujeres, se destaca junto con el impulso por la igualdad, el derecho a la diferencia. Esto supone afirmar una manera distinta de ver la realidad, de experienciarla desde un cuerpo, desde un lugar particular en el mundo. Dicha apelación a la diferencia constituye un mensaje hacia toda la sociedad, pues logran conjugar el poder y la diferencia, dado que "[l]a confrontación con el poder masculino ha enseñado a las mujeres a conocer cómo la diferencia se transforma en poder" (MELUCCI, 1984, p.138). Dicha diferencia además se articula con dimensiones específicas de la vida cotidiana que van desde el campo intelectual, la salud, la ocupación de casas, el trabajo, las cooperativas y el pacifismo (Marina BIANCHI y Maria MORMINO, 1984).

El análisis de los conflictos permite identificar acciones colectivas en formación, o bien su ausencia, su fragmentación, su cooptación. De esta última línea se desprende esta presentación, en la que se articulan las nociones propuestas por Adrián Scribano de geometrías corporales, gramáticas de las acciones y sensibilidades que forman parte de una Sociología de los cuerpos y las emociones (SCRIBANO, 2004; 2012) para describir e interpretar datos secundarios de Argentina en 2002, provenientes de Latinobarómetro.

\section{Las clases sociales y las relaciones de géneros en clave de geometrías y gramáticas}

La revisión precedente nos pone en la necesidad de explicitar algunas relaciones conceptuales que subyacen a las dimensiones aquí presentadas. Desde una Sociología de los cuerpos y las emociones (SCRIBANO, 2012) definimos a los agentes sociales como cuerpos que dialécticamente se configuran desde la socialización, la individuación y la subjetivación.

Los agentes sociales conocen el mundo a través de los cuerpos. Este conocimiento práctico, se configura porque sus estructuras cognitivas/afectivas resultan de haber incorporado las estructuras del mundo. Este comprensión práctica del mundo implica la configuración de las diferencias de géneros: en las formas de vestir, de andar, de mirar, de modo que las clasificaciones sociales se naturalizan como divisiones entre los cuerpos, sus hexis, sus disposiciones, sus esquemas de clasificación (Pierre BOURDIEU, 1999; 2007). Pero, además, la ubicación de los cuerpos en el mundo como ocupación de un lugar se plasma de manera estructural, relacional e histórica como posiciones y condiciones de clase (BOURDIEU, 2002).

La estructuración social del mundo en el que los cuerpos se ubican - y se diferencian entre sí por géneros y clases sociales- se sostiene desde hace 5 siglos bajo el modo de acumulación capitalista, el cual ha reconfigurado y reinscripto relaciones de dominación anteriores, tales como el patriarcado, la esclavitud. De allí que en la historia, la formación del trabajador asalariado haya tenido como contraparte necesaria en Europa, la "housewificación" de la mujer, mientras en las colonias americanas esclavos y esclavas provenientes de África eran explotados en las plantaciones de caña de azúcar (Silvia FEDERICI, 2010). Así pues, los cuerpos en el capitalismo pueden ser vistos en términos de 
clases sociales y géneros. Las clases no son infinitas ni las elabora el estadista. Son múltiples, y su construcción cotidiana, si bien puede cambiar, implica que son producto y productoras de una trama estructural que combina contingencias y trayectorias (BOURDIEU, 2002).

En este contexto, las clases sociales pueden ser identificadas a partir de tres dimensiones, en tanto conjunto de posiciones y disposiciones (SCRIBANO, 2010b): 1. la forma en que se da la expropiación de energías corporales y sociales, principalmente a través del trabajo, 2. la forma en que otro agente disfruta de dicha expropiación, y 3. los modos en que se concreta la desposesión de bienes comunes. La expropiación depende del lugar ocupado en el mercado de trabajo, desde donde se realiza un "embargo" de energías corporales (mientras dura el trabajo y más allá, porque tiene que dormir para trabajar, comer para trabajar, etc.) y, un mecanismo por el cual se sacan energías sociales, es decir, otros vínculos no mercantiles. Por las formas de expropiación se da un enclasamiento de prácticas que naturalizan los lugares y los modos en que se vive, sea un asalariado o bien un cuentapropista. El disfrute desde el otro, como segundo componente de las clases, se da en esa relación de expropiación de energías corporales y sociales, en el sentido en que Marx lo planteó en el marco de la relación capitalista-obrero. La desposesión común tiene que ver con las formas generales por las que el capital se apropia del agua, la tierra, entre otros, particularmente en el Sur Global.

Desde las posiciones y condiciones que forman una clase, los agentes aprehenden, elaboran y formatean sus estructuras cognitivo-afectivas, elaboran distintas valoraciones, se aprecian y son apreciados. Estas estructuras les permiten ocupar dichos lugares sociales y (re)producir las relaciones en que participan, de allí aquí que exista una estrecha relación entre las percepciones que forman las valoraciones y el conflicto.

En este marco, se han formulado dos categorías entrelazadas para entender desde una perspectiva estructuralista quiénes son los agentes que participan en un conflicto (SCRIBANO, 2004). La "geometría de los cuerpos" - resultante de la expropiación, disfrute del otro, desposesión de bienes comunes - consiste en la capacidad de disponer del propio cuerpo, y la "gramática de las acciones" remite a disponibilidad de su capacidad de acción. En el caso de conflictos laborales, la geometría corporal tiene que ver, por ejemplo, con cuánto pueden sostener las trabajadoras en una maquila, la explotación a la que son sometidas, cuánto pueden presionar, cuánto poder tienen para obtener un aumento de salario. La gramática, por su parte, tiene que ver con lo que los agentes pueden hacer a nivel social, según el contexto y las interacciones. Ambas categorías están atravesadas por la disponibilidad de acción que consiste en las energías corporales y sociales.

Si bien estas categorías permiten mapear los conflictos, de la misma relación entre geometrías y gramáticas se deriva la posibilidad de que el conflicto se neutralice, evite o desplace. Por ejemplo, si es injusto el pago por el trabajo realizado, pero los otros compañeros no tienen otra forma de conseguir ingresos, no se reclama nada por temor al despido. Precisamente, la propuesta del artículo es mapear las (des)conexiones entre estas categorías.

Como se presenta en el apartado 2.2., las geometrías corporales pueden ser identificadas a partir de variables que dan cuenta de condiciones materiales de vida, en tanto que las gramáticas se describen en función de prácticas o acciones realizadas de modo colectivo. En síntesis, si las geometrías refieren a posiciones y las gramáticas a capacidad de acción, entre ambas operan las formas de apreciar y clasificar, es decir, las percepciones que sostienen las valoraciones de un conflicto y también las emociones, las cuales, como se explicita a continuación, conforman las sensibilidades. 


\subsection{De los cuerpos/emociones y sensibilidades}

El aprendizaje social se realiza por y a través del cuerpo, que mantiene un lugar destacado para la afectividad, y las transacciones afectivas con el entorno. Al respecto los actos rituales dentro de los grupos, como las prácticas cotidianas de socialización, constituyen un proceso psicosomático que "se ejerce a menudo mediante la emoción y el sufrimiento, psicológico o incluso físico, en particular, el que se inflige inscribiendo signos distintivos, mutilaciones, escarificaciones o tatuajes, en la superficie misma del cuerpo" (BOURDIEU, 1999, p. 187). La relación estrecha que mantienen las tramas corporales con las emociones - construidas socialmente pero articuladas con procesos neurofisiológicos que también se construyen socialmente - (Gabriela VERGARA, 2014) evocan una forma particular de sentir el mundo, por el cual se producen naturalizaciones, acostumbramientos, formas de soportar las injusticias, la explotación, la dominación.

Como el mundo se conoce por el cuerpo, las emociones resultan de las relaciones dialécticas entre impresiones, percepciones y sensaciones (aquí cabe recordar que el agente tiene sensaciones respecto de su propio cuerpo construidas a partir del registro vivencial del mismo, por lo cual el fenómeno del miembro fantasma que interpreta Merleau Ponty puede ser pensado en referencia a una parte del cuerpo que aunque no esté, se siente debido a su presencia pasada).

Percepciones, emociones y sensaciones, constituyen a las sensibilidades, las cuales se articulan con las formas aceptadas y naturalizadas de interacción - sociabilidad - y con los sentidos de un yo que se relaciona con otros - vivencialidades. De este modo, el sistema capitalista puede articular las condiciones objetivas de vida como vivencias con lo socialmente aceptable, construyendo sensibilidades acordes a la desigualdad. Las sensibilidades se construyen, circulan y reproducen a partir de la implicación elíptica de tres procesos:

1) las conexiones - y desconexiones- entre afecciones individuales, percepciones sociales y relaciones de clase, etnia, género y edad; 2) las disposiciones y dispositivos clasificatorios respecto a lo que se presenta socialmente como "medio" "entorno" "naturaleza" y/o "planeta" y 3) el diseño de las formas tecnológicas de vehiculizar, transportar y dislocar el tiempo y el espacio (SCRIBANO, 2010a, p. 255).

En este artículo, nos centraremos particularmente en analizar el primer proceso, utilizando datos secundarios que pueden reinscribirse conceptualmente con lo aquí expuesto.

El interés por esta indagación tiene que ver con identificar la presencia de mecanismos que contribuyen a evitar el conflicto y dispositivos que regulan las sensaciones (y por lo tanto afectan las sensibilidades), permitiendo la continuidad estable del sistema.

La trama conceptual desarrollada precedentemente busca construir una forma de comprender que ciertas prácticas de los agentes sociales (mayor o menor participación política, mayor o menor involucramiento en las cuestiones barriales, laborales, ecológicas, entre otros) no se deben a una pura contingencia sino a la inscripción de dichos agentes en posiciones y condiciones de clase que, siguiendo a Scribano, se han definido aquí como geometría de los cuerpos y gramáticas de las acciones. Ahora bien, considerando el lugar de las emociones en la participación de los sujetos en los movimientos sociales ${ }^{6}$ (línea de estudio que sigue el creciente interés por las emociones en las ciencias sociales) y a una definición general de los agentes como cuerpos inescindidos de sus emociones, se articulan estas categorías con el estado de la sensibilidad.

\footnotetext{
${ }^{6}$ Alberto Melucci rescata del análisis de los movimientos sociales la reflexividad de las acciones de los sujetos, y a la vez "la comunicación que encuentra espacio, no sólo para lógica instrumental de la eficiencia, sino también para los sentimientos, incertidumbres y conflictos emocionales que siempre alimentan la acción humana" (1994, p. 138).
}

6 Revista Estudos Feministas, Florianópolis, 26(1): e43607 


\subsection{Sobre los datos secundarios}

En lo que sigue, se presentan las variables tomadas de Latinobarómetro, ${ }^{7}$ agrupadas de acuerdo con las categorías previamente desarrolladas:

- Geometrías corporales: Variables sociodemográficas (sexo, edad, estado civil, nivel educativo); Condición de ocupación (situación ocupacional, tipo de ocupación, principal ingreso familiar / desempleado en el hogar en últimos 6 meses); Propiedades, bienes y servicios (casa propia, segunda vivienda, auto, computadora, teléfono fijo, gastos en salud).

- Sensibilidades: Percepciones sobre situación personal y del país, y percepción de ingreso subjetivo (alcanza-no alcanza) (presentes); Sensación de preocupación sobre el empleo en próximos 12 meses / percepción de situación personal futura; Felicidad o satisfacción con la vida / percepción de problemas importantes en el país.

- Gramáticas de la acción: Mirar y esperar, Hacer algo para cambiar; Prácticas (firmar una petición, asistir a manifestaciones autorizadas, participar en saqueo, ocupar terreno, edificio o fábrica); otras prácticas (por el barrio, por el trabajo, por personas necesitadas, por lo ambiental)

De acuerdo con la información disponible en Latinobarómetro, las geometrías corporales se conforman a partir del tipo de ocupación del entrevistado - es la forma que adquiere la expropiación de energías en el trabajo, principalmente -, junto con variables sociodemográficas, posesión de bienes o servicios, y cercanía con el desempleo. Esto brinda un panorama de ciertas condiciones de vida que pueden dar cuenta de posiciones y condiciones sociales diferenciales. En Argentina, desde principios de este siglo se convive con las consecuencias del Programa de Ajuste Estructural que, entre otros aspectos, generó profundas transformaciones en el mercado laboral.

En cuanto a las sensibilidades, se seleccionaron variables vinculadas con la percepción presente de la situación familiar y del país, del futuro y preocupación por el empleo, junto con la felicidad y percepción de problemas. Las tensiones entre el presente y el futuro en Argelia (BOURDIEU, 2006), en un contexto de cambio de estructuras económicas, pusieron de manifiesto desajustes en las percepciones y sensaciones de los sujetos.

Por último, las gramáticas de la acción se relevan a partir de la selección de respuestas que confirman la realización de tales prácticas. Un grupo de variables tienen que ver con acciones de protestas, manifestaciones, mientras que el resto se vinculan con prácticas menos disruptivas como puede ser colaborar con dinero para el barrio. Junto a esto, se adicionaron dos variables que mapean una disposición a la acción (mirar y esperar o hacer algo para cambiar).

\section{Del trabajo al conflicto: análisis de dos grupos ocupacionales}

En este apartado se describen las variables agrupadas precedentemente según las categorías de geometrías corporales, sensibilidades y gramáticas de la acción, aplicando la herramienta análisis online de Latinobarómetro, disponible en su página web. En todos los casos, las tablas de contingencia elaboradas tienen como variables independientes al tipo de ocupación y género.

De la muestra para Argentina, de 1200 casos, el 44,3\% son activos. En los inactivos, se destaca "no trabaja-responsable de compras y cuidado", que está sobrerrepresentada

\footnotetext{
${ }^{7}$ Desde otro enfoque teórico, María Luz Morán y Marisa Revilla (2008) utilizan Latinobarómetro para indagar la participación de las mujeres en acciones colectivas.
} 
en las mujeres. ${ }^{8}$ Casi el $40 \%$ de las mujeres encuestadas es ama de casa, y de las restantes, el principal grupo trabaja (sin considerar a las retiradas-jubiladas) como independiente y empleada en empresa privada. En las categorías asalariado en empresa pública, retiradopensionado y estudiante, no hay diferencias. Según el género, el tipo de ocupación de activos presenta la distribución:

- Empleados (en sector público y privado): dentro de las mujeres activas, el $56,6 \%$ se desempeña bajo esta modalidad, al igual que el $38,8 \%$ de los varones activos.

- Trabajador por cuenta propia, ambulante no profesional: en esta categoría se ubica el $24,3 \%$ de mujeres y $30,7 \%$ de varones.

- Dueño de negocio: se registraron $8,3 \%$ mujeres y $16 \%$ varones.

- Otras categorías (agricultor, alto ejecutivo y profesional asalariado): 2,9\% mujeres y $5,8 \%$ varones.

- Ejecutivo de mando medio: $2 \%$ mujeres y $4.5 \%$ varones. varones.

- Profesional (doctores, abogados, contadores, etc.): $5.9 \%$ en mujeres y $4.2 \%$ en

De acuerdo con estos datos, se destaca, por un lado, la categoría empleado (que abarca al sector privado y público) en la cual las mujeres superan en 18 puntos a los varones. En el resto, hay mayor presencia masculina, con excepción de los profesionales. En Ejecutivo de mando medio se observa segregación vertical. ${ }^{9}$

Los empleados y cuentapropistas no profesionales reúnen la mayor cantidad de casos. Los segundos (excluyen a dueño de negocio y profesional) parecen mostrar una de las secuelas de la aplicación del Programa de Ajuste Estructural vinculado a la precariedad, pérdida de beneficios sociales y la lógica del 'sálvese si puede' (Alfredo PUCCIARELLI, 1998). Según las características de esta muestra, el cuentapropismo triplica al empleo en el sector público.

\subsection{Las geometrías corporales}

La edad, el estado civil y el nivel educativo dan cuenta de ciertas condiciones de vida que se cualifican según la inserción laboral.

Tabla 1 - Edades según ocupación y género

\begin{tabular}{|c|c|c|c|c|}
\hline \multirow{2}{*}{ Edad } & \multicolumn{2}{|c|}{ Empleados/as } & \multicolumn{2}{c|}{ Cuentapropistas } \\
\cline { 2 - 5 } & $\begin{array}{c}\text { varones } \\
(\mathrm{n}=131)\end{array}$ & $\begin{array}{c}\text { mujeres } \\
(\mathrm{n}=110)\end{array}$ & $\begin{array}{c}\text { varones } \\
(\mathrm{n}=103)\end{array}$ & $\begin{array}{c}\text { mujeres } \\
(\mathrm{n}=47)\end{array}$ \\
\hline $15-25$ & 23 & 21,2 & 20,1 & 16,7 \\
\hline $26-40$ & 40,9 & 43,9 & 37,2 & 21,2 \\
\hline $41-60$ & 32 & 30,3 & 33,4 & 50 \\
\hline $61 y+$ & 4,1 & 4,6 & 9,3 & 12 \\
\hline
\end{tabular}

Fuente: elaboración propia en base a Latinobarómetro (2002).

\footnotetext{
${ }^{8}$ Según datos de CEPAL, hacia 2005, solo 1 de cada 5 hogares en Latinoamérica mantenía el patrón de varón-proveedor, mujer-ama de casa (Irma ARRIAGADA, 2007).

${ }^{9}$ Lo mismo sucede en alto ejecutivo, donde directamente no se registran casos en mujeres.
}

8 Revista Estudos Feministas, Florianópolis, 26(1): e43607 
El primer grupo (Tabla 1) presenta valores más elevados en el rango de edades de 26-40, sin diferencias significativas según el género. En los cuentapropistas, una de cada dos mujeres tiene entre 41-60 años, mientras que el 12\% tiene más de 61 (cifra que triplica al mismo grupo de las asalariadas). Los varones, por su parte, muestran una distribución similar entre los 26-60 años. Los cuentapropistas en general presentan un mayor envejecimiento, pero más en las mujeres.

El estado civil indica que el $60 \%$ de los varones asalariados y el $45 \%$ de las mujeres son casados/convivientes. La brecha entre géneros aumenta en separadas/divorciadas/ viudas: $4,2 \%$ son varones y $23,6 \%$ son mujeres. ${ }^{10}$ Poco más de un tercio de las mujeres cuentapropistas es casada-conviviente, y otro tercio está separada/divorciada/viuda (superando en 15 puntos a las empleadas). En los varones, predominan los casados/ convivientes, aunque aumentan los separados.

Las empleadas poseen un nivel educativo más alto que los varones y las cuentapropistas. En asalariados, la mitad de los varones $(53,5 \%)$ tiene hasta secundario incompleto, ${ }^{11}$ mientras que las mujeres, casi un tercio $(33,2 \%)$. Como contrapartida en el siguiente nivel educativo, el $60 \%$ de las mujeres tiene secundaria completa y superior incompleto (los varones alcanzan el $43,5 \%$ ). Esta tendencia a favor de las mujeres se mantiene también en el nivel superior completo ( $3 \%$ varones, $6,9 \%$ mujeres). En cambio, el $66 \%$ de las cuentapropistas tiene hasta secundario incompleto y el resto, secundario completo. No se registran casos de terciario-universitario completo, lo que sí se da en el $2 \%$ de los varones.

En los asalariados, el $73,4 \%$ de los varones y el $53,7 \%$ de las mujeres se identifican como principal ingreso, el resto de casos femeninos (46,3\%) seguramente forman parte de hogares de doble ingreso. En los cuentapropistas, el $62 \%$ de las mujeres es principal ingreso ( $9 \%$ más que empleadas), mientras que lo es el $75,7 \%$ de los varones. En este caso, las diferencias más acentuadas se registran entre las mujeres, y quienes presentan mayor porcentaje de separadas.

Tabla 2. Bienes y servicios en los hogares ${ }^{12}$

\begin{tabular}{|l|l|l|l|l|}
\hline \multirow{2}{*}{ Bienes / servicios que se tienen } & \multicolumn{2}{|c|}{ Empleados } & \multicolumn{2}{c|}{ Cuentapropistas } \\
\cline { 2 - 5 } & Varones & Mujeres & Varones & Mujeres \\
\hline casa propia & $62,6 \%$ & $\mathbf{7 3 , 5} \%$ & $\mathbf{7 3 , 3} \%$ & $62,9 \%$ \\
\hline segunda propiedad o casa & $\mathbf{3 , 2} \%$ & $2,8 \%$ & $\mathbf{6 , 6} \%$ & $2,4 \%$ \\
\hline Auto & $31,5 \%$ & $\mathbf{3 8} \%$ & $\mathbf{4 0 , 4} \%$ & $17,5 \%$ \\
\hline telefonia fija & $65,3 \%$ & $\mathbf{7 2} \%$ & $53,9 \%$ & $\mathbf{6 1} \%$ \\
\hline Computadora & $23,9 \%$ & $\mathbf{3 8 , 1} \%$ & $\mathbf{2 5 , 2} \%$ & $20,3 \%$ \\
\hline
\end{tabular}

Fuente: elaboración propia en base a Latinobarómetro (2002).

\footnotetext{
10 Dada la edad de estas mujeres activas, se infiere que el mayor porcentaje de esta categoría sean las separadas y divorciadas.

${ }^{11}$ Incluye primaria incompleta, completa y secundario incompleto.

${ }^{12}$ En las columnas se presentan los valores de las frecuencias positivas, es decir, quienes tienen vivienda, auto etc. Para facilitar la presentación de los datos, no se incluyen los valores negativos que completan el $100 \%$ de cada columna de varones o mujeres.
} 
La situación general del hogar se identificó con la presencia de al menos un adulto desempleado en últimos 12 meses: el 55\% de los varones, y el 62,6\% de las mujeres empleadas respondió afirmativamente. La cercanía del cuentapropismo con el desempleo es mayor en los varones (72\%) que en las mujeres (65\%), y respecto de los empleados (superan en 17 puntos).

La posesión de algunos bienes y servicios se observa en la Tabla 2.

La carencia de una casa propia afecta al $40 \%$ aproximadamente de varones empleados y mujeres cuentapropistas. Sus pares varones parecen estar relativamente mejor, incluso por destacarse en tener una segunda propiedad y auto.

En las asalariadas se observa una mayor dotación de bienes y servicios respecto de los varones del mismo grupo y mucho más que las cuentapropistas, donde sólo en telefonía fija las mujeres superan a sus pares masculinos. La posesión de auto se destaca en varones cuentapropistas (posiblemente, dedicados al transporte de personas vía taxis o remises, una de las opciones de trabajo que encontraron los indemnizados tras quedar sin empleo en los 90') quienes, como contrapartida, presentan un menor acceso a telefonía fija. Tener una computadora puede asociarse al grupo de mujeres con mayor nivel educativo.

En cuanto al gasto en salud, 3 de cada 10 asalariados no tienen cobertura (el $31 \%$ en los varones y el $25.5 \%$ en las mujeres), lo cual indica precariedad en la relación laboral. En los cuentapropistas se reduce el acceso a este servicio. El $72 \%$ de los varones y el $60 \%$ de las mujeres no tienen cobertura, pero los superan gastos privados ( $12 \%$ a $8 \%$ ) y públicos $(27,8 \%$ a $19,40 \%)$.

En términos de las geometrías corporales, se observa que:

- dentro de los asalariados, las mujeres cuentan con mejor nivel educativo y recursos materiales en su vida (casa, automóvil, telefonía, computadora, cobertura de salud). Un grupo de estas mujeres (las que no son principal ingreso) posiblemente tengan algunas ventajas por ser segunda proveedora del hogar, a diferencia de aquellas que contribuyen directamente al sostenimiento del hogar, como las divorciadas. Los empleados muestran menor nivel educativo, menos bienes y servicios aunque menor exposición al desempleo, comparado con sus pares femeninas.

- en el cuentapropismo, las mujeres tienen mayor edad, menor nivel educativo formal; hay más divorciadas y más jefas de hogar y, poseen menos recursos. Los varones presentan más dotación de bienes (incluso que los empleados), aunque en ambos aumenta la falta de cobertura de salud y la cercanía con el desempleo.

Desde estas posiciones y condiciones diversas cuando se articula ocupación y género es que se inscriben e interpretan algunos componentes de las sensibilidades.

\subsection{Las sensibilidades desde el empleo y el cuentapropismo}

En este apartado se presentan los datos considerando: a) percepciones de ingreso subjetivo (alcanza-no alcanza), situación personal y del país, presentes; b) percepción de situación familiar futura y sensación de preocupación sobre el empleo en próximos 12 meses; c) felicidad y percepción de problemas.

a) Percepciones en tiempo presente

El ingreso subjetivo es una valoración de prácticas concretas en relación con los recursos monetarios del hogar. Si bien por sí sola esta variable no indica riqueza (alcanza) o pobreza (no alcanza), dado que un hogar puede tener altos niveles de ingreso/consumo y percibir que no les alcanza el dinero, en el contexto de otras variables analizadas en este artículo aporta un mapeo de cómo era percibida la situación presente de los entrevistados.

Como se observa en la Tabla 3., a 7 de cada 10 asalariados y al $65 \%$ de las mujeres no le alcanzan los ingresos (recordemos que el 55\% afirmó tener un desocupado en el hogar). 
Tabla 3. Comparación de percepciones de ingreso y situaciones personal/país

\begin{tabular}{|c|c|c|c|c|c|c|c|}
\hline \multicolumn{8}{|c|}{ Empleados y empleadas } \\
\hline \multirow[t]{2}{*}{$\begin{array}{l}\text { Categoria } \\
\mathrm{s}\end{array}$} & \multicolumn{2}{|c|}{ Ingreso subjetivo } & \multirow[t]{2}{*}{$\begin{array}{l}\text { Categoria } \\
\mathrm{s}\end{array}$} & \multicolumn{2}{|c|}{$\begin{array}{c}\text { Situación personal } \\
\text { actual }\end{array}$} & \multicolumn{2}{|c|}{$\begin{array}{l}\text { Situación pais } \\
\text { actual }\end{array}$} \\
\hline & $\begin{array}{l}\text { varone } \\
s\end{array}$ & $\begin{array}{l}\text { mujere } \\
s\end{array}$ & & varones & $\begin{array}{l}\text { muje } \\
\text { res }\end{array}$ & varones & mujeres \\
\hline Alcanza & $29,3 \%$ & $35,1 \%$ & $\begin{array}{l}\text { Muy } \\
\text { buena } \\
\text {-buena }\end{array}$ & 14,9 & $\begin{array}{r}11,3 \\
\%\end{array}$ & $1,9 \%$ & - \\
\hline- & - & - & Regular & 52, & $\begin{array}{r}51,1 \\
\%\end{array}$ & $4,9 \%$ & $6,8 \%$ \\
\hline $\begin{array}{l}\text { No } \\
\text { alcanza }\end{array}$ & $70,6 \%$ & $64,9 \%$ & $\begin{array}{l}\text { Mala-muy } \\
\text { mala }\end{array}$ & 3. & $\begin{array}{r}37,6 \\
\%\end{array}$ & $93,2 \%$ & $93,2 \%$ \\
\hline \multicolumn{8}{|c|}{ Cuentapropistas } \\
\hline \multirow[t]{2}{*}{$\begin{array}{l}\text { Categoría } \\
8\end{array}$} & \multicolumn{2}{|c|}{ Ingreso subjetivo } & \multirow[t]{2}{*}{$\begin{array}{l}\text { Categoría } \\
\mathrm{s}\end{array}$} & \multicolumn{2}{|c|}{$\begin{array}{c}\text { Situación personal } \\
\text { actual }\end{array}$} & \multicolumn{2}{|c|}{$\begin{array}{c}\text { Situación pais } \\
\text { actual }\end{array}$} \\
\hline & $\begin{array}{r}\text { varone } \\
s\end{array}$ & $\begin{array}{r}\text { mujere } \\
s\end{array}$ & & varones & mujeres & varones & mujeres \\
\hline Alcanza & $24,4 \%$ & $15,2 \%$ & $\begin{array}{l}\text { Muy } \\
\text { buena } \\
\text {-buena }\end{array}$ & $6,4 \%$ & $6,8 \%$ & $0,7 \%$ & - \\
\hline- & & & Regular & $38,8 \%$ & $23,3 \%$ & $5,9 \%$ & $7,6 \%$ \\
\hline $\begin{array}{l}\text { No } \\
\text { alcanza }\end{array}$ & $75,6 \%$ & $84,8 \%$ & $\begin{array}{l}\text { Mala- } \\
\text { muy mala }\end{array}$ & $54,8 \%$ & $69,9 \%$ & $93,4 \%$ & $92,3 \%$ \\
\hline
\end{tabular}

Fuente: elaboración propia en base a Latinobarómetro (2002).

La situación económica personal actual se califica como muy buena-buena por el 14,9\% de varones y el $11,3 \%$ de mujeres. En mala-muy mala se ubica el $33 \%$ de los varones y el $37.6 \%$ de las mujeres, por lo cual el principal porcentaje ( $50 \%$ aproximadamente) se registra en regular. Si se comparan ambas variables el porcentaje de no alcanza duplica al de situación mala/muy mala. Dadas las características de las geometrías corporales de estos empleados, puede interpretarse que aunque el dinero no alcance, se cuenta con recursos para hacer frente a la situación actual, por lo cual puede entenderse que se perciba principalmente como regular. En relación con la situación del país, se observa que la categoría mala-muy mala triplica a la situación personal, lo cual indica la lógica del "no me va tan mal, al país le va peor". No se observan diferencias significativas entre géneros.

La situación es diferente en los cuentapropistas. El dinero no alcanza para el $85 \%$ de las mujeres, de las cuales el $70 \%$ califica como mala a la situación económica personal actual. A nivel país, la valoración es similar, no solo entre géneros, sino entre éstos y el grupo de asalariados, solo que en los cuentapropistas la distancia entre una percepción y otra se acorta ("a mí me va mal y al país muy mal") y más aún en las mujeres (92\%-70\%). Es decir que 
las mujeres cuentapropistas tienen una percepción presente más negativa que el resto sobre sus ingresos y la situación del país.

b) percepción de situación familiar futura y sensación de preocupación sobre el empleo en próximos 12 meses

Si indagamos a futuro, la situación económica personal de los asalariados se percibe mejor en $25,6 \%$ varones y $21,7 \%$ mujeres. En igual situación hay más mujeres $(36,8 \%)$ que varones, y en peor situación se invierte (46,1\%, varones y $41,5 \%$ mujeres). Casi el $90 \%$ de los encuestados en ambos géneros tiene preocupación de quedar sin trabajo en los próximos 12 meses. Aquí, puede advertirse cierta desconexión entre esta preocupación que duplica la percepción negativa de la situación futura y a las propias experiencias cercanas del desempleo.

La situación futura en los cuentapropistas se percibe de manera similar en términos porcentuales. Casi la mitad (48\%) de las mujeres de modo similar a los varones (43\%) avizoran empeoramiento. Sin embargo, un cuarto de los encuestados espera una mejora, lo cual se contrasta con el bajo porcentaje (7\%) de quienes ven la situación actual como buena-muy buena.

Si se tiene en cuenta que sólo cerca del 7\% valora la situación presente como buena, la mejoría a futuro opera como un mecanismo de soportabilidad, tanto en varones y mujeres. Esto se ve con mayor claridad si se compara con las percepciones de los asalariados, quienes mantienen una distancia similar entre la percepción presente-futura. En condiciones de cuentapropismo hay una percepción más negativa pero a la vez mayor esperanza de mejora. En cuanto a la situación de desempleo, la mayor preocupación se da en las mujeres $(93,5 \%)$. c) Felicidad y percepción de problemas

¿Cómo se sienten los asalariados en esta situación? La única pregunta en 2002 sobre la satisfacción o la felicidad muestra que 6 de cada 10 mujeres $(62,3 \%$ ) y 1 de cada 2 varones $(51,7 \%)$ está feliz. En los cuentapropistas de modo similar, 6 de cada 10 varones y mujeres, están felices.

Si se analizan los datos sólo considerando la felicidad, se podría decir que no hay variación por clase y género. Ahora bien, si se inscribe este dato en el marco de las variables que se proponen en este artículo, se entiende que la felicidad de una mujer cuentapropista jefa de hogar, con secundario incompleto, sin cobertura médica no es de la misma naturaleza que la de una asalariada en empresa pública que no es principal ingreso en su hogar.

Además es interesante ver cómo convive la felicidad con la percepción de problemas. El desempleo es identificado por el $29,5 \%$ de los varones y el $27,1 \%$ de las mujeres (pese a que 9 de cada 10 asalariados están preocupados). Bajos salarios preocupan a menos del $10 \%$ de los encuestados y casi por igual a varones y mujeres: $7.8 \%$ y $6.8 \%$ respectivamente.

La corrupción es más importante para varones $(21,1 \%)$ que para mujeres $(17,5 \%)$, mientras que la pobreza ( $11,7 \%$ contra $7,8 \%$ ) y la educación $(9,1 \%$ y 5,65 , respectivamente), predominan en las mujeres.

En el caso de los cuentapropistas, se destaca la desocupación/desempleo en ambos géneros $(29,2 \%$ en varones y $29,7 \%$ en mujeres), siendo apenas un tercio a lo registrado como preocupación de que este fenómeno los afecte directamente. Otra vez aparece una distancia entre situaciones cercanas y otras que aparecen con un menor grado de afectación. El segundo problema con similar intensidad en ambos sexos es la corrupción (15,3\% en varones, $14,1 \%$ en mujeres), aunque con menor intensidad que en los asalariados. Los bajos salarios preocupan al $12,7 \%$ de varones y al $11,5 \%$ de mujeres, valores que casi duplican a los empleados. La pobreza tiene una leve diferencia a favor de las mujeres $(11,4 \%)$ sobre los varones $(7,3 \%$ ) al igual que en la educación (10,6\% en mujeres y $5,9 \%$ en varones). 
Es interesante advertir que para las cuentapropistas la educación como problema aparece con igual porcentaje que las asalariadas, solo que las primeras tienen un menor nivel educativo.

Por otra parte se advierte cómo las percepciones tienen que ver con la posición y condición y el género. Los salarios preocupan a quienes padecen más del desempleo y viven en situación de que los ingresos no alcancen. La corrupción preocupa más a quienes tienen un empleo estable. En ambos grupos, la pobreza y la educación registran un mayor porcentaje en las mujeres.

Antes de pasar a las gramáticas, cabe considerar que en ambos grupos de una lista de 19 problemas ${ }^{13}$ se han identificado los 5 principales. El resto, obtiene cerca del $30 \%$. El eje de empleo/pobreza/salarios articula entonces el 50\% aproximadamente de todos los problemas percibidos.

\subsection{Las gramáticas de la acción}

En este apartado se presentan primero las disposiciones a la acción (mirar y esperar, ${ }^{14}$ hacer algo para que cosas cambien ${ }^{15}$ ), luego las prácticas en acciones colectivas en general y, por último la modalidad de participación sobre temáticas particulares.

Los valores obtenidos en ambos grupos de ocupados y en ambos géneros no presentan diferencias significativas. ${ }^{16}$ Aproximadamente la mitad acuerda con mirar $y$ esperar, aunque aumenta la disposición a hacer algo hasta el 80\%.

Tabla 4. Acciones realizadas ${ }^{17}$

\begin{tabular}{|l|l|l|r|r|}
\hline \multirow{2}{*}{ Acciones realizadas } & \multicolumn{2}{c|}{ Empleados } & \multicolumn{2}{c|}{ Cuentapropistas } \\
\cline { 2 - 5 } & Varones & Mujeres & Varones & Mujeres \\
\hline Firmar petición & $24 \%$ & $\mathbf{3 5 , 6} \%$ & $21,2 \%$ & $23,6 \%$ \\
\hline Asistir a manifestaciones autorizadas & $19,1 \%$ & $\mathbf{2 3 , 3} \%$ & $16,5 \%$ & $19 \%$ \\
\hline Participar en saqueo & - & - & $2,1 \%$ & - \\
\hline Ocupar un terreno, fábrica o edificio & $1,7 \%$ & - & $1,1 \%$ & $4,5 \%$ \\
\hline
\end{tabular}

Fuente: elaboración propia en base a Latinobarómetro (2002).

\footnotetext{
${ }^{13}$ Entre ellos se puede mencionar terrorismo/ violencia política/ guerrilla; falta de oportunidades para la juventud, violación de los DD.HH, problemas de vivienda/ habitación, consumo de drogas, discriminación racial, entre otros. Si se comparan desde 2004-2013 los problemas más importantes, se advierte sin mayores diferencias de género que, mientras la desocupación desciende, aumenta la delincuencia/seguridad pública, sobre todo a partir de 2009.

${ }^{14}$ La pregunta del cuestionario es: "¿Está muy de acuerdo, de acuerdo, en desacuerdo o muy en desacuerdo con las siguientes afirmaciones que le voy a leer?: Entre elección y elección lo único que puede hacer alguien como yo es mirar y esperar."

${ }^{15}$ La pregunta del cuestionario es: "¿Está muy de acuerdo, de acuerdo, en desacuerdo o muy en desacuerdo con las siguientes afirmaciones que le voy a leer?: Entre elección y elección la gente como yo debe hacer algo para que las cosas que no me gustan cambien."

${ }^{16}$ Los valores obtenidos en asalariados, son: $46.4 \%$ en mujeres y $52,1 \%$ en varones acuerdan mirar y esperar. En cuentapropistas, $55 \%$ en ambos géneros. En hacer algo, los varones asalariados y cuentapropistas acuerdan en el $85 \%$; mientras que en las mujeres asalariadas y cuentapropistas, alcanza el $78 \%$.

${ }^{17}$ En la tabla se presentan por filas sólo los datos correspondientes a esa categoría. No totalizan el 100\%, dado que el resto de las respuestas corresponden a nunca lo haría y podría realizarlo.
} 
Estas disposiciones a la acción se articulan con prácticas concretas:

Las cifras presentadas por filas indican que casi tres cuartas partes de los entrevistados se inclinan por la inacción (nunca lo haría o, podría). El valor más elevado que se registra inter e intra géneros en las empleadas que firmaron petición es mucho más bajo que el registrado en su disposición a hacer algo (78\%) o el desacuerdo con mirar y esperar (50\%). En saqueo y ocupación de terreno se obtuvo para las mujeres el $99 \%$ de respuestas en la categoría nunca lo haría y, esta negativa se encontró en la mitad de las mujeres para asistir a manifestaciones.

Las cuentapropistas superan levemente, excepto en saqueos, a los varones. Comparados con los asalariados, se observa más allá del género que en estos sujetos hay menos participación en peticiones y manifestaciones (en las asalariadas, era del $35 \%$ y23\%), y mayor en saqueos y ocupaciones, en particular las mujeres. En asalariados, sólo se registró el 1,7\% en varones, y ningún caso en saqueo.

Las desconexiones entre disposiciones y acciones emergen y resultan del proceso de configuración de las geometrías corporales con las sensibilidades a partir de las formas en que se perciben las situaciones personales, de los otros, del país. Llama la atención que las cuentapropistas, estando en condiciones más vulnerables y percibiendo una situación de modo más negativo -con una cercanía entre la situación personal y la del país- no tengan mayor participación que el resto. La firma de una petición parece indicar cierta gramática pulcra, no directamente vinculante -no se pone el cuerpo como en una manifestación- que guarda correspondencia con las geometrías de las empleadas. De todos modos, en clave de género, esta participación supone un tiempo que se debe quitar al trabajo reproductivo del hogar, que queda invisibilizado en este registro.

En lo que sigue, se presentan los datos de otro tipo de acciones concretas, de carácter menos disruptivo como puede ser un saqueo:

Una primera lectura de la Tabla 5. permite advertir quiénes y en qué tipos de acciones no se han involucrado. Si se comparan las categorías ninguna, en varones y mujeres en las cuatro acciones, se advierte que lo ambiental presenta el porcentaje más elevado de inacción, dentro del cual se destacan los cuentapropistas de ambos géneros. La ayuda a personas necesitadas es lo que más moviliza a ambos grupos, dado que registra el menor porcentaje $(60 \%)$ en la opción de respuesta ninguna. Aunque la pobreza se percibe como problema solo para el $11 \%$ (en las mujeres, porque en varones es menor), estas prácticas pueden dar cuenta del solidarismo como una de las formas que traman las relaciones con los otros. La problemática barrial moviliza más a las empleadas, quienes presentan el porcentaje más bajo en ninguna. El barrio como lugar próximo de vida parece ser un ámbito donde estas mujeres tienen un leve protagonismo por encima de los varones.

En las cuestiones laborales, hay más inacción en los cuentapropistas, seguramente, debido a la modalidad de trabajo que realizan. Cabe destacar que el porcentaje más elevado de acciones concretas efectuadas (que se registra en mujeres asalariadas, modalidad trabajo para cuestiones laborales, $21 \%$ ), es inferior a la firma de peticiones. Los reducidos porcentajes de acciones por temáticas específicas y la dispar participación en manifestaciones, petitorios, entre otras, alerta sobre la linealidad de pensar que cuando hay crisis, la gente se moviliza.

Los datos analizados desde la perspectiva teórica señalada párrafos arriba nos permiten reflexionar acerca de cierto estado del "individualismo" en las sociedades latinoamericanas del Siglo XXI. Para ello, podemos pensar a modo de "pistas conceptuales" en fenómenos que ya fueron identificados desde un lugar subjetivista como de uno objetivista. En el primer caso nos referimos a Georg Simmel, quien identifica dos individualismos, uno promovido por el liberalismo en contra de las ataduras que en el siglo 
Tabla 5. Tipos de acciones según problemáticas específicas

\begin{tabular}{|c|c|c|c|c|c|}
\hline \multirow{2}{*}{ Ámbito de acciones } & & \multicolumn{2}{|c|}{ Empleados } & \multicolumn{2}{|c|}{ Cuentapropistas } \\
\hline & $\begin{array}{c}\text { Tipo } \\
\text { de acciones }\end{array}$ & Varones & Mujeres & Varones & Mujeres \\
\hline \multirow{4}{*}{$\begin{array}{l}\text { Hacer algo por la } \\
\text { comunidad o el barrio }\end{array}$} & Trabajo & $9,4 \%$ & $14,68 \%$ & $13,6 \%$ & $8,5 \%$ \\
\hline & Reuniones & $0,8 \%$ & $2,76 \%$ & $2,9 \%$ & $2,14 \%$ \\
\hline & Dinero & $10 \%$ & $13,76 \%$ & $9,7 \%$ & $14,9 \%$ \\
\hline & Ninguna & $79,8 \%$ & $68,8 \%$ & $73,8 \%$ & $74,46 \%$ \\
\hline \multirow{4}{*}{$\begin{array}{l}\text { Acciones para mejorar } \\
\text { condiciones } \\
\text { laborales o actividad en } \\
\text { que se desempeña }\end{array}$} & Trabajo & $17,69 \%$ & $21,10 \%$ & $16 \%$ & $8,50 \%$ \\
\hline & Reuniones & $1,54 \%$ & $=$ & $1 \%$ & - \\
\hline & Dinero & $11,54 \%$ & $6,42 \%$ & $6 \%$ & $6,40 \%$ \\
\hline & Ninguna & $69,23 \%$ & $72,48 \%$ & $77 \%$ & $85,10 \%$ \\
\hline \multirow{4}{*}{$\begin{array}{l}\text { Acciones para ayudar } \\
\text { a personas necesitadas }\end{array}$} & Trabajo & $17,69 \%$ & $16,82 \%$ & $18 \%$ & $19,15 \%$ \\
\hline & Reuniones & $13,85 \%$ & $17,76 \%$ & $17 \%$ & $8,52 \%$ \\
\hline & Dinero & $8,46 \%$ & $5,61 \%$ & $5 \%$ & $12,76 \%$ \\
\hline & Ninguna & $60,00 \%$ & $59,81 \%$ & $60 \%$ & $59,57 \%$ \\
\hline \multirow{4}{*}{$\begin{array}{l}\text { Acciones para mejorar } \\
\text { el medio ambiente }\end{array}$} & Trabajo & 10,94 & 15,74 & $10 \%$ & $8,50 \%$ \\
\hline & Reuniones & - & . & $=$ & . \\
\hline & Dinero & 4,69 & 4,63 & $2 \%$ & $4,25 \%$ \\
\hline & Ninguna & 84,38 & 79,63 & $88 \%$ & $87,25 \%$ \\
\hline
\end{tabular}

Fuente: elaboración propia en base a Latinobarómetro (2002).

XVIII constreñían al individuo en términos políticos, religiosos, corporativos, etc., y el otro emparentado con el romanticismo alemán, que buscaba la singularidad de lo humano. Las grandes ciudades operan como teatros donde combaten, o podrían conciliarse, estos dos individualismos. En este contexto, en la gran ciudad, Simmel advierte se configura un "hombre blasé", quien se caracteriza por haberse

vuelto insensible a las diferencias entre las cosas; no que no las perciba, ni que sea estúpido, sino que la significación y el valor de esas diferencias, y por tanto de las cosas mismas, él los percibe como negligibles. Los objetos se le aparecen en una tonalidad uniformemente sosa y gris; ninguno se juzga digno de preferencia (SIMMEL, 1986, p. 4).

Esta actitud es el reflejo subjetivo de la economía monetaria en su apogeo y podría derivar en desconexiones respecto de los demás. 
Por otra parte, Jürgen Habermas identificó privatismo en tanto "indiferencia política unida con el interés dominante de la carrera, el tiempo libre y el consumo" (HABERMAS, 1999, p.74) que podría operar como sustento de ciertas prácticas epitomizadas bajo el "mientras me va bien, lo que les pasa a los otros no me preocupa". De igual modo, dichos fenómenos se reconfiguran en los cruces de clases y géneros. En el párrafo que sigue esbozamos algunas interpretaciones provisorias de estos fenómenos.

\section{A modo de conclusión}

A decir de Bourdieu, la estadística es una de las herramientas para la ruptura con el sentido común. El análisis propuesto constituye una primera fase deco-construcción de datos y una red categorías conceptuales que permiten comprender las experiencias de quienes participan o no en acciones colectivas, a fin de dar respuestas tentativas a la cuestión de porqué estando en condiciones de protestar, varones y mujeres asalariados o cuentapropistas parecen ser más esperantes que protestantes. Por ello, se considera que si las emociones intervienen en las acciones colectivas, dado que son constitutivas de los agentes sociales, inciden también desde las estructuras cognitivo-afectivas en los procesos previos a la emergencia del conflicto, (des)articulando, (des)conectando y dando sentido a prácticas y gramáticas diversas.

Por otra parte, la selección de los tipos de ocupación comparados -empleados y cuentapropistas- no pretende construir clases de papel, sensu Bourdieu, sino enfatizar cómo desde distintas posiciones y condiciones sociales según la forma que adquiere la expropiación de energías corporales, se percibe/siente el mundo y se interviene en él. Otra lectura no menos interesante sería recorrer un camino similar considerando a las personas encuestadas por Latinobarómetro como responsables de las compras y cuidado.

Como un camino en construcción, lo presentado aquí no pretende agotar ni monopolizar las relaciones entre variables y conceptos, sino generar opciones que permitan incrementar las posibilidades de comprensión de la estructuración capitalista actual. Tampoco pretende cuestionar la vasta producción académica en torno a las asambleas, marchas, saqueos, empresas recuperadas que se sucedieron en ese período en Argentina, aunque sí alertar respecto de la otra cara del fenómeno, de quienes vivenciaron la crisis como testigos o espectadores.

En términos generales, el análisis/interpretación precedente permite identificar al menos dos fenómenos que interrogan y desafían los horizontes de la acción colectiva:

- por un lado, se observa inacción en quienes tienen certezas en sus condiciones de vida (sobre todo en empleados y empleadas), lo que les permite percibir su situación como regular, mientras al resto le va mal. Esta lógica afirma un lugar de autoprotección que inhabilita prácticas por los otros, aunque se esté preocupado por el desempleo, pero relativamente feliz con la vida. De aquí se desprende un individualismo que convive en algunos casos con cierto solidarismo. Mientras cada cual busca salvarse como puede, en el mejor de los casos, las ayudas no solo brindan recompensas emocionales, sino que además permiten marcar distancias (otros están peor, y más que esto no puedo hacer). En esto se destaca una trama entre mujeres jóvenes, con mayor nivel educativo, casadas, empleadas que cuentan con más recursos y perciben la situación regular, aunque se involucran en algunas acciones, tales como los petitorios y trabajo en cuestiones vinculadas al medio ambiente, al barrio, a personas necesitadas y a lo laboral. Las certezas en las condiciones materiales de vida pueden otorgar una mayor capacidad de acción pese a que la felicidad, las preocupaciones y los problemas puedan ser similares - al menos estadísticamente hablando - a otros y otras. Aunque no son la mayoría, en las gramáticas se destacan por un interés en hacer algo, más allá de su hogar y su ámbito laboral. 
- por otra parte, se advierte cierta lógica de inacción en la vulnerabilidad puesto que, de los datos analizados se destacan cómo las mujeres cuentapropistas tienen mayor edad, están menos educadas, la mayoría es divorciada y presentan un escenario de mayor vulnerabilidad, que se combina con una peor percepción de su situación cercana a la del país. Sin embargo, esta sintonía de percepciones inscriptas en condiciones materiales de vida limitadas, en convivencia con cierto grado de felicidad, no genera mayores acciones o involucramientos colectivos, sino todo contrario. En este caso, el individualismo pareciera estar articulado con otro tipo de emociones sociales que pueden encontrar en la espera de una situación futura mejor, vestigios de impotencia y resignación, las cuales pueden operar haciendo soportable la vida cotidiana.

Las trayectorias, las biografías de luchas colectivas pueden haber dejado semillas de parálisis crecientes. Pero, además, un conjunto de medidas implementadas desde esa época en adelante también pueden haber contribuido a aplacar el camino del conflicto, a través de la cooperativización de la pobreza (como lo muestra el epígrafe), de la implementación de planes sociales y del consumo como la forma por excelencia de ser parte de la sociedad, aunque ya no se esté ocupado.

\section{Referencias}

ANDÚJAR, Andrea. "Pariendo resistencias: las mujeres piqueteras de Cutral Co y Plaza Huincul (1996)". En: BRAVO, María Celia, GIL LOZANO, Fernanda, PITA, Valeria (comps.). Historia de luchas, resistencias y representaciones. Mujeres en la Argentina, siglos XIX y XX. Tucumán: EDUNT, 2007. p. 151-181.

ARRIAGADA, Irma. "Familias latinoamericanas: cambiantes, diversas y desiguales". Papeles de Población, n. 53, julio-septiembre, p. 9-22, Toluca, 2007.

BENSKI, Tova \& LANGMAN, Lauren. The effects of affects: The place of emotions in the mobilizations of 2011. Current Sociology, v. 61, p. 525-540, July, 2013.

BIANCHI, Marina y MORMINO, Maria. "Militanti di se stesse. II movimento delle donne a Milano". In: Alberto Melucci (ed). Altri codici: aree di movimento nella metropoli. Bologna: Societá Editrice II Mulino.1984. p. 127-173.

BOLTANSKI, Lùc y CHIAPELLO, Eve. El nuevo espíritu del capitalismo. Barcelona: Akal, 2002.

BOURDIEU, Pierre. Meditaciones pascalianas. Barcelona: Anagrama, 1999.

BOURDIEU, Pierre. "Condición de clase y posición de clase". Revista Colombiana de Sociología, v. VII, n. 1, p.119-141, 2002.

BOURDIEU, Pierre. Argelia '60. Buenos Aires: Siglo XXI Editores, 2006.

BOURDIEU, Pierre. EI Sentido práctico. Buenos Aires: Siglo XXI Editores, 2007.

CID AGUAYO, Beatriz. "Maternizando lo político: mujeres y género en el movimiento sindical de la Industria Salmonera en Chile". Revista Estudos Feministas, Florianópolis, v. 20, n. 1, p. 189-207, 2012. Disponible en: <http://www.scielo.br/scielo.php?script=sci_arttext\&pid=S0104026X2012000100011 \&lng=en\&nrm=iso >. Acceso el: 18 noviembre 2017.

DALLA COSTA, Mariarosa. Capitalismo e riproduzione. Relazione presentata al Seminario "Women's Unpaid Labor and the World System" organizzato dalla Japan Foundation l'8 aprile 1994 a Tokyo all'interno dell' "European Women's Study Tour for Environmental Issues" sponsorizzato dalla Japan Foundation.

DI MARCO, Graciela. Los movimientos de mujeres en la Argentina y la emergencia del pueblo feminista. La Aljaba, v.14, p. 51-67, 2010.

FEDERICI, Silvia. El Calibán y la bruja. Madrid: Traficantes de sueños, 2010.

FEDERICl, Silvia. Revolución en punto cero. Madrid: Traficantes de sueños, 2013. 
HABERMAS, Jürgen. Problemas de legitimación en el capitalismo tardío. Madrid: Cátedra, 1999.

HARVEY, David. "The 'new' imperialism: accumulation by dispossession". Socialist Register, n. 40, p. 63-87, 2004

KAPLAN, Temma. "Conciencia femenina y acción colectiva. El caso de Barcelona, 19101918". En: AMELANG, James S. y NASH, Mary (coords.). Historia y género: las mujeres en la Europa moderna y contemporánea. València: Edicions Alfons el Magnànim, Institució Valenciana d'Estudis i Investigació, 1990. p. 267-296.

LOBATO, Mirta. Historia de las trabajadoras en la Argentina (1869-1960). Buenos Aires: Edhasa, 2007.

MELUCCI, Alberto. “¿Qué hay de nuevo en los nuevos movimientos sociales?”. En: LARAÑA, Enrique, y GUSFIELD, Joseph (edits.). Los nuevos movimientos sociales. Madrid: CIS, 1994. p. $119-150$.

MORÁN, María Luz y REVILLA, Marisa. "Mujeres y política en América Latina: más allá de la participación política formal". En: PRIETO, Mercedes (edit.). Mujeres y escenarios ciudadanos. Quito: FLACSO, 2008. p. 159-194.

NECOECHEA, Gerardo. "Nosotros somos oprimidas, esposas de obreros: mujeres y política en Río Blanco". Estudios de Culturas Contemporáneas, año 2, n. 6, p. 175-201, 1989.

PUCCIARELLI, Alfredo. "¿Crisis o decadencia? Hipótesis sobre el significado de algunas transformaciones recientes de la sociedad argentina". Revista Sociedad de la Facultad de Ciencias Sociales, UBA, n. 12/13, p. 5-36, 1998.

SCRIBANO, Adrián. Conflicto y estructuración social: una propuesta para su análisis. En: ZEBALLOS, Eliseo; TAVARES DO SANTOS, José Vicente; SALINAS FIGUEREDO, Darío. (Edits). América Latina: hacia una nueva alternativa de desarrollo. Arequipa: Editorial Universidad Nacional de San Agustín, 2004. p. 54-68.

SCRIBANO, Adrián. "Las sensibilidades prohibidas: el epílogo de un libro sobre la transformación social". En: SCRIBANO, Adrián y LISDERO, Pedro (comps.). Sensibilidades en juego: miradas múltiples desde los estudios sociales de los cuerpos y las emociones. Córdoba. CEACONICET, 2010a. p. 246-257.

SCRIBANO, Adrián. "Tesis 1: Colonia, conocimiento(s) y Teorías sociales del Sur". Boletín Onteaiken, n. 10, p. 1-22, 2010b.

SCRIBANO, Adrián. "Sociología de los cuerpos/emociones". Relaces, n. 10, p. 93-113, diciembre-marzo, 2012.

SCRIBANO, Adrián. "Cuerpos y emociones en El Capital". Nómadas, n. 39, p. 29-45, july/dec, 2013.

SCRIBANO, Adrián y ARTESSE, Matías. "Emociones y acciones colectivas: un bosquejo preliminar de su situación hoy". En: CERVIO, Ana (comp.). Las tramas del sentir. Ensayos desde una sociología de los cuerpos y las emociones. Buenos Aires: ESE Editora, 2012. p. 85-105.

SCRIBANO, Adrián y BOITO, Eugenia (comps.). El purgatorio que no fue. Acciones profanas entre la esperanza y la soportabilidad. Buenos Aires: CICCUS, 2010.

SEVESO ZANÍN, Emilio y VERGARA MATTAR, Gabriela (2012). "En el cerco. Los cuerpos precarios en la ciudad de Córdoba tras la crisis argentina de 2001". Papeles del CEIC, 79, marzo, Centro de Estudios sobre la Identidad Colectiva, País Vasco, p. 1-38.

SIMMEL, Georg. "Las grandes ciudades y la vida del espíritu". Cuadernos Políticos, n. 45, p. 5-10, 2006.

VEENHOVEN, Ruut. "Return of inequality in modern society?" Journal of Happiness Studies, v. 6, p. 457-487, 2005.

VERGARA, Gabriela. "Emociones, cuerpos y residuos: un análisis de la soportabilidad social". Revista Brasileira de Sociologia da Emoção, n. 37, p. 43-58, abril, 2014.

18 Revista Estudos Feministas, Florianópolis, 26(1): e43607 
VERGARA, Gabriela y D'AMICO, Marcelo. "Crisis e incertidumbre: un análisis de las experiencias colectivas en Córdoba y Villa María desde los cuerpos y las emociones". En: SCRIBANO, Adrián y BOITO, Eugenia (comps.). El purgatorio que no fue. Acciones profanas entre la esperanza y la soportabilidad. Buenos Aires: CICCUS, 2010. p. 53-81.

[Recebido em 14/03/2016,

reapresentado em 20/02/2017

e aprovado em 11/04/2017]

Bodies, Sensibilities and Collective Action (Argentina, 2002)

Abstract: This paper analyzes the relationships between living conditions, sensibilities and actions, to understand the ways in which the conflict may (or may not) emerge. Secondary data from Latinobarometro are used, in 2002, the year of the last major crisis in Argentina. Employees and selfemployed, women and men are compared. Analysis shows that there is inaction under two different but complementary approaches: individualism/solidarism and individualism/bearability. This quantitative approach is not intended to exhaust the proposed concepts and relationships between variables, but encourage the possibilities of understanding of the current capitalist structuration. Key words: Bodies; Sensibilities; Conflict; Gender; Labor

Gabriela del Valle Vergara (gabivergaramattar@gmail.com) es investigadora adjunta de Conicet con lugar de trabajo en la Secretaría de Investigación y Extensión del Instituto Académico Pedagógico de Ciencias Sociales de la Universidad Nacional de Villa María. Doctora en Ciencias Sociales. Mgter. en Ciencia Sociales. Lic. en Sociología. Investigadora del Centro de Investigaciones y Estudios Sociológicos (CIES). Investigadora del Grupo de Estudios sobre Subjetividades y Conflicto (GESSYCO). Integrante del Programa de Estudios sobre Acción Colectiva y Conflicto Social del CIECS-CONICET. 